\title{
Estendendo o Conhecimento sobre a Família Her-Receptores para o Fator de Crescimento Epidérmico e seus ligantes às Malignidades Hematológicas

\author{
Extending Knowledge on Her-Family Receptors for Epidermal Growth Factor \\ and their Ligands to Hematological Malignancies
}

Claudia Sondermann Freitas*

\section{Resumo}

Os receptores da família HER (ErbB) são fundamentais para o desenvolvimento de diversos órgãos e sistemas. Embora sua presença em tecidos adultos seja ubíqua, sua superativação ou desregulação está relacionada a um mau prognóstico para diversos tipos de tumores. Curiosamente, HER e seus ligantes são ausentes nas células maduras do sangue, o que sugere que sua expressão deva ser "desligada" em algum momento do desenvolvimento hematopoético normal. Diversos relatos, no entanto, têm evidenciado a presença de transcritos e/ou proteínas para esses receptores e seus ligantes em leucemias e linfomas, sugerindo que, também nestas patologias, as novas drogas que vêm sendo desenvolvidas para inibir HER em outros tipos de câncer possam contribuir para o controle do crescimento celular. Largamente conhecidos por suas funções sinalizadoras transmembranosas, os receptores HER foram recentemente observados em núcleos celulares, sugerindo possibilidades adicionais de compreensão e controle da sinalização por esses receptores. Essa presença foi proposta como fator prognóstico para câncer de mama, o que poderá vir a se estender também a malignidades hematológicas.

Palavras-chave: EGF, HER, Câncer, Hematopoese, Inibidores de tirosina-quinases, Tirfostinas

*PhD, Pesquisadora do Programa de Onco-hematologia, MEDEX, Coordenadoria de Pesquisa, Instituto Nacional de Câncer Endereço para correspondência: Claudia Sondermann Freitas. Rua André Cavalcante 37 - $6^{\circ}$ andar - Rio de Janeiro (RJ), Brasil - CEP: $20230-130$. E-mail: csondermannf@inca.gov.br 


\section{A FAMÍLIA HER EM CÂNCER}

Os receptores HER1, HER2, HER3 e HER4, também chamados ErbB, (proteínas tirosina-quinases transmembranosas que ligam o fator de crescimento epidérmico - EGF - e outras moléculas assemelhadas) são fundamentais para o desenvolvimento de diversos órgãos e sistemas. Embora sua expressão em tecidos adultos seja ubíqua, sua superativação está relacionada a um mau prognóstico para diversos tipos de tumores ${ }^{1}$, esteja ela relacionada à amplificação dos genes HER, a mutaçôes nesses genes ${ }^{2,3}$, ou ainda a uma simples desregulação do programa de diferenciação para cada tipo celular. Quando ativos, esses receptores unem-se em dímeros, se transfosforilam, e passam a ser capazes de transduzir sinais intracelulares que podem afetar o crescimento celular, a inibição da apoptose, a migração e a invasividade, a angiogênese, entre os processos que levam à progressão de tumores malignos ${ }^{4,5}$.

HER1 (EGFR, ErbB1) se acha superexpresso em virtualmente todas as malignidades epiteliais (pulmão, cólon, próstata, mama), representando um prognóstico ainda pior do que a superexpressão de HER2 ${ }^{6}$. No entanto, a simples superexpressão de HER1 não transforma células, já que o dímero HER1:1 só é capaz de se transfosforilar quando a ele se acopla um de seus ligantes extracelulares (EGF ou outros membros dessa família de fatores de crescimento). Esses fatores podem ser produzidos pelos tumores ou por células não-malignas adjacentes, gerando uma indução ao crescimento autócrina ou justácrina. Já HER2, para o qual não foi descrito ainda um ligante extracelular, pode espontaneamente formar dímeros, uma característica conferida pela estrutura peculiar de sua porção extracelular. Assim, o dímero HER2:2 pode se transfosforilar independentemente da ausência de ligante, estimulando a transformação morfológica e o crescimento celular, seja em sua forma mutada (o oncogene neu, formando homodímeros neu:neu), seja na sua forma não mutada. De fato, HER2 não mutado é capaz de formar heterodímeros com HER1 constituindo a quinase ativa HER1:2, assim fosforilando HER1 mesmo na ausência de seus ligantes extracelulares ${ }^{6}$.

Além das bem descritas funções sinalizadoras desses receptores como moléculas transmembranosas, funções ainda pouco definidas relacionadas à sua presença diretamente no núcleo celular vêm sendo evidenciadas ${ }^{7,8,9}$. Em tumores de mama, foi relatado o valor prognóstico de HER1 nuclear, inversamente relacionado à sobrevivência dos pacientes ${ }^{10}$.

\section{DIVERSIFICAÇÃO DE RECEPTORES E VIAS DE SINALIZAÇÃO}

A síntese e o processamento dos transcritos e das proteínas para os receptores HER estão sujeitos a mecanismos de regulação ainda não completamente compreendidos $^{11,12,13}$. Sabe-se que os peptídeos que compõem essa família de receptores são codificados por diferentes genes (em humanos, EGFR - HER1, cromossoma 7; HER2, cromossoma 17; HER3, cromossoma 12; HER4, cromossoma 2). Dois ou mais membros da família HER podem ser expressos por um mesmo tipo celular, sugerindo que seus produtos não são simplesmente redundantes, mas exercem uma diversificação de funçôes sinalizadoras ${ }^{6}$.

Cada um desses genes pode ainda gerar vários produtos, como, por exemplo, o gene para HER1, que gera peptídeos de pesos moleculares 7.5, 44, 69, 72, $77,110,115,134$, e $170 \mathrm{kDas}$, alguns truncados em regiōes $\mathrm{N}$-terminais (extracelulares), outros em regiōes C-terminais (intracelulares, contendo a região com atividade de quinase e os sítios de transfosforilação), vide as seqüências depositadas em bancos de dados (NCBI/NIH/USA; Uniprot/EBI/UK; Swiss-prot/ ExPaSy/ISB/SW). Pouco se sabe a respeito da função desses peptídeos "variantes". No caso de HER4, por exemplo, foram descritas duas variantes que diferem na porção justamembranosa e se expressam de forma tecido-específica, apresentando algum grau de diferença funcional ${ }^{12,14}$.

Além das variantes, foram descritas também formas mutantes de HER, associadas à sua ativação aberrante em tumores ${ }^{15-21}$.

Cada um dos peptídeos HER pode se associar em homo ou heterodímeros, mesmo quando um só membro do par encontra seu ligante ${ }^{6}$, gerando uma diversidade de receptores. Assim, HER2 figura como um excelente "parceiro" para transfosforilaçōes, atuando como coreceptor para outros membros da família, que podem ou não estar acoplados aos seus ligantes extracelulares $^{22,23}$. Por exemplo, embora o HER3 não possua atividade intrínseca de tirosina-quinase, sendo seu dímero HER3:3 inativo, ele pode formar heterodímeros e ser transfosforilado por HER4 ou HER2 quando "acionado" pelos fatores de crescimento ligantes de HER3 (Neuregulinas e Heregulinas) ${ }^{4,12,23}$. Ademais, a formação de heterodímeros com HER2 aumenta a afinidade de todos os outros membros da família a seus respectivos ligantes ${ }^{24}$. No caso de HER3, a dimerização com HER2 aumenta dramaticamente sua afinidade por Neuregulinas e torna sua porção C-terminal inativa como quinase extremamente ativa como transdutora de 
sinais mitogênicos, por oferecer muitos sítios adicionais de fosforilação ${ }^{25}$. HER3 é também um ótimo parceiro, por não sofrer degradação intralisossomal, sendo continuamente reciclado e refosforilado pelo HER2 superexpresso, e contribuindo ativamente para a transformação celular ${ }^{26}$.

A diversificação de funções de HER pode se dar também pela utilização de diferentes vias intracelulares de sinalização ${ }^{26,27,28}$. Assim, heterodímeros são mais potentes e mitogênicos, fornecendo resíduos adicionais de fosfotirosina, que podem acoplar diferentes moléculas intermediárias na transdução de sinais ${ }^{1,26}$. Por exemplo, todos os dímeros de HER ativam a via de Ras/MAPK, enquanto apenas alguns deles são capazes de ativar a via de PI3-K/Akt. Enquanto PI3-K se acopla diretamente a HER3 e HER4, seu acoplamento a HER1 ou HER2 depende de uma proteína intermediária Cbl23, que também modula a atividade dessas quinases pela regulação diferencial de sua degradação ${ }^{29}$.

Como um mecanismo adicional de diversificação, cada um desses dímeros pode ainda aceitar a ligação de mais de um fator de crescimento da família do EGF, diferentes ligantes estimulando diferentes mecanismos de transdução de sinais. Assim, a região C-terminal de HER1, que contém os sítios de autofosforilação, é essencial à atividade mitogênica induzida pela ligação de Anfiregulina, mas é perfeitamente dispensável para a mitogênese induzida por EGF, sendo ambos os fatores ligantes de HER $1^{30}$. Ligantes de baixa afinidade pelos receptores podem inclusive modificar o tempo do estímulo, resultando em um aumento da meia-vida do receptor e modificando as vias pelas quais este será degradado ${ }^{1}$.

Além de ter sua degradação modificada pelos ligantes, os receptores HER podem também ter sua expressão regulada por eles, resultando assim em um conjunto de ligantes, receptores, transdutores de sinal e fatores de transcrição, bastante específico para uma dada situação biológica, o que torna o controle de seus efeitos extremamente complexo ${ }^{1,27}$.

\section{FORMAS TRUNCADAS DE RECEPTORES HER}

Formas truncadas para a porção intracelular, C-terminal, desses receptores, foram descritas em tumores sólidos e linhagens celulares malignas ${ }^{13,31,32}$, e suas possíveis funçōes permanecem por ser esclarecidas. Sabe-se que a deleção de alguns sítios de transfosforilação C-terminais não impede a transdução de sinais por esses receptores, através da fosforilação de sítios alternativos ${ }^{33}$. Além disso, a remoção da cauda citoplasmática de HER1 resulta em um receptor truncado com alta propensão a se ligar a HER2, que potencializa a resposta mitogênica a EGF. Foi sugerido assim que a presença dessa cauda funcionaria mais propriamente como um bloqueador das interaçôes de HER1 com HER2 na ausência de ligante extracelular, impedindo a ativação espontânea e "indesejada" ${ }^{30}$.

A propensão de HER2 em se associar a homodímeros ou a outros peptídeos HER sugere que existam, na fisiologia normal, mecanismos que evitem a formação espontânea de homodímeros de HER2 ${ }^{1}$. De fato, Herstatina revelou-se como um fragmento da porção extracelular, N-terminal, de HER2, que, solúvel, cumpre esse papel de "inibidor natural". Além de inibir a dimerização e a auto-ativação de HER2, é capaz de ligar-se a HER1, HER2, HER4, deltaHER1 e, surpreendentemente, até a IGF-1R, mas não a HER3 ou FGFR- $3^{34}$, inibindo também a indução de expressão de HER3 e HER4 pelo HER2 superexpresso, a transativação de HER3 por HER2 e a ativação de HER1 de forma ligante-dependente em células que o superexpressam ${ }^{35,36}$.

Já ao gene para HER3, correspondem quatro pequenos transcritos alternativos relacionados à porção extracelular, inicialmente observados em carcinomas de ovário, mas também presentes em tecidos normais humanos ${ }^{37}$. Para um dos peptídeos solúveis de HER3 (p85), pôde-se demonstrar uma função inibitória à ativação pela competição com o ligante Heregulina ${ }^{38}$.

Também para HER1 de humanos, camundongos, ratos e aves, foram observados transcritos curtos provenientes de regiōes $\mathrm{N}$-terminais, cujos produtos funcionam como inibidores de transformação celular dependente de ligante ${ }^{39,40}$. Pequenos fragmentos inibidores vêm sendo estudados como candidatos para o desenvolvimento de novas terapias anticâncer ${ }^{36}$.

\section{PEPTÍDEOS LIGANTES DA FAMÍLIA HER}

No que diz respeito aos ligantes extracelulares (fatores de crescimento da família do EGF), semelhante diversidade de transcritos e proteínas foi observada, a partir de diferentes genes ${ }^{41}$. Acham-se descritos em organismos superiores pelo menos 12 membros dessa família: os ligantes de HER1 (EGF, TGF alfa, HB-EGF, Anfiregulina, Epiregulina, Betacelulina), e os de HER3 e HER4 (as Neuregulinas, produtos do processamento alternativo dos transcritos do gene NRG-1, que incluem: as Heregulinas, o fator de diferenciação neuronal - NDF, o fator de crescimento da glia - GGF; o produto do gene NRG-2; e NTAK - ativador de quinases de origens neural e tímica) $)^{1,22,42}$.

Alguns desses ligantes utilizam um único receptor, por exemplo, o TGF alfa e a Anfiregulina, que só ligam 
HER1, ou as Neuregulinas 3 e 4, que só ligam HER4. Outros utilizam mais de um receptor, como o HB-EGF, a Betacelulina e a Epiregulina, que ligam tanto HER1 como HER4, ou as Neuregulinas 1 e 2, que ligam tanto HER3 como HER $4^{43}$.

Alguns deles podem ainda ser ativos sob a forma de seu precursor transmembranoso, não sendo secretado sob a forma solúvel e ativando células contíguas de forma justácrina, pelo contato célula-célula ${ }^{44,45}$. Ademais, podem exercer função autócrina, no caso de uma mesma célula produzir tanto receptores quanto ligantes, alimentando seu próprio crescimento ${ }^{46}$. Diversos tumores mostram crescimento autócrino pela produção de HER e de seus ligantes, por exemplo, tumores do trato aerodigestivo superior, em que HER são sintetizados simultaneamente a EGF e TGF alfa ${ }^{47}$.

\section{INIBIDORES SINTÉTICOS DE HER}

Diversas abordagens terapêuticas vêm sendo desenvolvidas tendo como alvo essas proteínas, tanto pelo uso de anticorpos "humanizados" dirigidos às quinases de membrana ${ }^{5,23,48-50}$ como pela síntese de pequenas moléculas capazes de entrar nas células e assim inibir tanto as quinases de membrana como as intracitoplasmáticas ${ }^{4,51}$. Dentre essas pequenas moléculas, acham-se as tirfostinas, sintetizadas com o objetivo de inibir seletivamente tirosina-quinases como, por exemplo, as tirfostinas AG1478 e RG13022, específicas para HER ${ }^{52}$. Outros inibidores dirigidos a HER1 ou 2 incluem o ZD1839 (Gefitinib, Iressa, Astra-Zeneca Pharmaceuticals, DE, USA), o OSI-774 (Tarceva, Erlotinib HCl, OSI Pharmaceuticals/ Genentech Inc., CA, USA), o PKI-166 (Novartis Pharma GA, Basel, SW), além dos inibidores pan-HER CI-1033 (Pfizer Inc., NY, USA) e GW572016

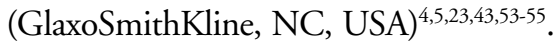

Estudos sobre as possíveis causas de escape ao tratamento com essas novas drogas vêm sendo desenvolvidos, sejam relacionados às mutaçōes nos sítios de ação dos inibidores, de forma a conferir resistência a esses agentes, ou a deficiências na ação de proteínas supressoras de tumor endógenas, sejam relacionados à expressão concomitante, por células tumorais, de mais de uma quinase, ou ainda à transmodulação, efetuada pelos mediadores intracelulares de transdução de sinais, de uso comum a diversas vias ${ }^{19,56-60}$. Conhecer esses mecanismos permitirá, em futuro próximo, que pacientes sejam selecionados de forma a aumentar suas chances de benefício clínico pelo uso dessas novas drogas, que vêm sendo testadas também em associação a outros quimioterápicos, em diversos tipos de tumores sólidos e leucemias ${ }^{4,5,23,51,54,55,60-62}$.

\section{HER E SEUS LIGANTES NO DESENVOLVIMENTO HEMATOPOÉTICO NORMAL OU MALIGNO}

É no mínimo curioso que, dentre os tecidos adultos normais, HER e seus ligantes estejam ausentes apenas nas células maduras do sangue ${ }^{41}$. Isso sugere que, em algum momento durante o desenvolvimento hematopoético, sua expressão deva ser "desligada", ou seja, negativamente modulada. Ainda mais curioso é o fato de que pouco se saiba sobre a "desregulação" desse programa em processos malignos do sistema hematopoético. Até porque essa família de receptores teve seu primeiro membro descrito quando da descoberta de seu oncogene análogo viral (v-ErbB) na transformação leucêmica de progenitores eritróides em aves, sendo posteriormente descrito o receptor celular endógeno (c-ErbB, ou HER2) e suas funçōes no desenvolvimento eritróide normal ${ }^{63-66}$.

$\mathrm{Na}$ literatura, alguns relatos apontam para a presença desses receptores ou seus ligantes em células malignas do sangue, de linhagens diversas. Por exemplo, a expressão de HER2 foi observada em pacientes com leucemia linfoblástica aguda e leucemia mielóide crônica quando em crise blástica $\mathrm{B}^{67}$, além da superexpressão de HER2 em um subgrupo de pacientes portadores de mieloma múltiplo ${ }^{68}$. Mais recentemente, foram observados peptídeos truncados de HER1 em células da medula óssea de pacientes portadores de leucemia mielóide crônica ${ }^{69}$. Além disso, a expressão de transcritos para um membro da família de ligantes, o HB-EGF, foi descrita em leucemias mielóides agudas ${ }^{70}$.

Mahtouk et al. ${ }^{62}$ relataram a expressão de vários membros dessa família de receptores e seus ligantes em linhagens celulares derivadas de mielomas múltiplos, mantidas em cultivo pela adição de IL-6, assim como em células de pacientes portadores desta patologia, leucemizadas ou não. Nesse estudo, utilizando o inibidor pan-HER PD169540 in vitro, para a inibição de crescimento e/ou estímulo à morte por apoptose, de células mononucleares da medula ou do sangue de oito pacientes, apenas dois não foram respondedores. Esses resultados sugerem que as novas drogas inibidoras de HER, que vêm sendo desenvolvidas para uso em cânceres epiteliais, poderão futuramente ser aplicadas também ao controle de crescimento celular em malignidades hematológicas, como o mieloma múltiplo, seja em associação ao bloqueio dos receptores para IL-6, visando à inibição do crescimento celular ou à indução de morte celular por Dexametasona.

Uma das linhas de pesquisa presentemente desenvolvidas na CPQ/INCA diz respeito à busca de 
transcritos e proteínas para HER e seus ligantes em células hematopoéticas malignas de linhagens diversas. Trabalhos anteriores, utilizando a tirfostina AG1478, específica para HER1, em cultivo de órgãos (timos fetais de camundongos), apontaram para um papel de HER1 e do precursor de EGF no desenvolvimento linfóide $\mathrm{T}$ normal ${ }^{71,72}$. Isso levou a pesquisar a modulação negativa da expressão dessas moléculas no processo de maturação de células do sangue, e a relação de desvios desse programa de diferenciação com processos malignos do desenvolvimento hematopoético. Inicialmente, foram observados transcritos para o precursor de EGF nas três linhagens linfóides $\mathrm{T}$ malignas murinas e uma humana testadas ${ }^{73}$, e pôde-se imunoprecipitar, utilizando anticorpos comerciais anti-EGF humano, peptídeos relacionados ao EGF contidos nos extratos da linhagem leucêmica $T$ humana CCRF-CEM (resultados não publicados).

Mais recentemente, foram observados transcritos para HER1 em linhagens celulares leucêmicas mantidas em cultivo, incluindo uma eritróide (K562), e 3 de 4 linhagens linfóides B estabelecidas a partir de linfomas de Burkitt (Daudi, Ramos, e Raji), além de transcritos para HER4 nesta última, e para os ligantes EGF, HBEGF ou TGF alfa, em todas as 14 linhagens testadas ${ }^{74,75}$.

Acha-se em fase inicial de implantação um projeto de autoria própria, dentro do Convênio Científico INCA-Fiocruz, no qual pretende-se, com a colaboração de Dr. Marcelo Pelajo (Fiocruz), analisar a possível presença de HER no núcleo de células malignas do sangue, utilizando ensaios morfológicos de imunomarcaçôes para esses receptores, com leitura em microscopia confocal, e em protocolos especiais que empregam permeabilização com detergentes em doses crescentes, visando à exposição de antígenos nucleares. Além disso, serão efetuados estudos bioquímicos, como análise por immunoblotting dessas proteínas em frações subcelulares, e estudos fisiológicos, empregando cultivos celulares sob observação ao microscópio, buscando a visualização do fluxo ou gradiente desses ligantes e receptores dentro das células. Contando com a interação de grupos clínicos que manifestem interesse na área, pretende-se poder acrescentar dados quanto à possível contribuição do crescimento HER-dependente para a evolução de doenças malignas do sangue.

\section{REFERÊNCIAS}

1. Marmor MD, Kochupurakkal BS, Yarden Y. Signal transduction and oncogenesis by ErbB/HER receptors. Int J Radiat Oncol Biol Phys. 2004;58:903-13.

2. Lassus H, Sihto H, Leminem A, Joensuu H, Isola J, Nupponen NN, et al. Gene amplification, mutation, and protein expression of EGFR and mutations of ERBB2 in serous ovarian carcinoma. J Mol Med. 2006;84:671-81.

3. Sihto H, Puputti M, Pulli L, Tynninen O, Koskinen W, Aaltonen L-M, et al. Epidermal growth factor receptor domain II, IV, and kinase domain mutations in human solid tumors. J Mol Med. 2005;83:976-83.

4. Vlahovic G, Crawford J. Activation of tyrosine kinases in cancer. Oncologist. 2003;8:531-38.

5. Herbst RS. Review of epidermal growth factor biology. Int J Radiat Oncol Biol Phys. 2004;59:21-26.

6. Earp HS, Dawson TL, Li X, Yu H. Heterodimerization and functional interaction between EGF receptor family members: a new signaling paradigm with implication for breast cancer research. Breast Cancer Res Treat. 1995;35:115-32.

7. Lin S-Y, Makino K, Xia W, Matin A, Wen A, Kwong KY, et al. Nuclear localization of EGF receptor and its potential role as a transcription factor. Nat Cell Biol. 2001;3:802-808.

8. Carpenter G. Nuclear localization and possible functions of receptor tyrosine kinases. Curr Opin Cell Biol. 2003;15:143-48.

9. Lo H-W, Xia W, Wei Y, Ali-Seyed M, Huang SF, Hung MC. Novel prognostic value of nuclear epidermal growth factor receptor in breast cancer. Cancer Res. 2005;65:338-48.

10. Lo H-W, Hung M-C. Nuclear EGFR signaling network in cancers: linking EGFR pathway to cell cycle progression, nitric oxide pathway and patient survival. Brit J Cancer. 2006;94:184-88.

11. Haley JD, Waterfield MD. Contributory effects of de novo transcription and premature transcript termination in the regulation of human epidermal growth factor receptor prooncogene RNA synthesis. J Biol Chem. 1991;266:1746-753.

12. Elenius K, Corfas G, Paul S, Choi CJ, Rio C, Plowman GD, et al. A novel juxtamembrane domain isoform of HER4/ErbB4. J Biol Chem. 1997;272:26761-6768.

13. Reiter JL, Threadgill DW, Eley GD, Strunk KE, Danielsen AJ, Sinclair CS, et al. Comparative genomic sequence analysis and isolation of human and mouse alternative EGFR transcripts encoding truncated receptor isoforms. Genomics. 2001;71:1-20.

14. Kainulainen V, Sundvall M, Maatta JA, Santiestevan E, Klagsburn M, Elenius K. A natural ErbB4 isoform that does not activate phosphoinositide 3-kinase mediates proliferation but not survival or chemotaxis. J Biol Chem. 2000;275:8641-649.

15. Bargmann CI, Hung MC, Weinberg RA. Multiple independent activations of the neu oncogene by a point mutation altering the transmembrane domain of $\mathrm{p} 185$. Cell. 1986;45:649-57.

16. Haley JD, Hsuan JJ, Waterfield MD. Analysis of mammalian fibroblast transformation by normal and mutated human EGF receptors. Oncogene. 1989;4:273-83.

17. Wong AJ, Ruppert JM, Bigner SH, Grzeschik CH, 
Humphrey PA, Bigner DS, et al. Structural alterations of the epidermal growth factor receptor gene in human gliomas. Proc Natl Acad Sci USA. 1992;89:2965-969.

18. Moscatello DK, Holgado-Madruga M, Godwin AK, Ramirez G, Gunn G, Zoltick PW, et al. Frequent expression of a mutant epidermal growth factor receptor in multiple human tumors. Cancer Res. 1995;55:5536-539.

19. Voldborg BR, Damstrup L, Spang-Thomsen M, Poulsen HS. Epidermal growth factor receptor (EGFR) and EGFR mutations, function and possible role in clinical trials. Ann Oncol. 1997;8:1197-206.

20. Pedersen MW, Meltorn M, Damstrup L, Poulsen HS. The type III epidermal growth factor receptor mutation. Biological significance and potential target for anti-cancer therapy. Ann Oncol. 2001;12:745-60.

21. Lorimer IA. Mutant epidermal growth factor receptors as targets for cancer therapy. Curr Cancer Drug Targets. 2002;2:91-102.

22. Yarden $Y$, Sliwkowiski MX. Untangling the ErbB signaling network. Nat Rev Mol Cell Biol. 2001;2:127-37.

23. Mass RD. The HER receptor family: a rich target for therapeutic development. Int J Radiat Oncol Biol Phys. 2004;58:932-40.

24. Jones JT, Akita RW, Sliwkowiski MX. Binding specificities and affinities of egf domains for ErbB receptors. FEBS Lett. 1999;447:227-31.

25. Citri A, Skaria KB, Yarden Y. The deaf and the dumb: the biology of ErbB2 and ErbB3. Exp Cell Res. 2003;284:54-65.

26. Waterman H, Alroy I, Strano S, Seger R, Yarden Y. The Cterminus of the kinase-defective neuregulin receptor ErbB3 confers mitogenic superiority and dictates endocytic routing. EMBO J. 1999;18:3348-358.

27. Riese II DJ, Kim ED, Elenius K, Buckley S, Klagsbrun M, Plowman GD, et al. The epidermal growth factor receptor couples transforming growth factor-a, heparin-binding epidermal growth factor-like factor, and amphiregulin to $\mathrm{Neu}$, ErbB-3, and ErbB-4. J Biol Chem. 1996;271:20047-20052.

28. Sweeney C, Carraway III. Ligand discrimination by ErbB receptors: differential signaling through differential phosphorylation site usage. Oncogene. 2000;19:5568-573.

29. Marmor MD, Yarden Y. Role of protein ubiquitylation in regulating encocytosis of receptor tyrosine kinases. Oncogene. 2004;23:2057-2070.

30. Wong L, Deb TB, Thompson SA, Wells A, Johnson GR. A differential requirement for the $\mathrm{COOH}$-terminal region of the epidermal growth factor (EGF) receptor in amphiregulin and EGF mitogenic signaling. J Biol Chem. 1999;274:8900-909.

31. Ullrich A, Coussens L, Hayflick JS, Dull TJ, Gray A, Tam AW, et al. Human epidermal growth factor receptor cDNA sequence and aberrant expression of the amplified gene in A431 epidermoid carcinoma cells. Nature. 1984;309:418-25.

32. Ilekis JV, Gariti J, Niedeerberger C, Scoccia B. Expression of a truncated epidermal growth factor receptor-like protein (TEGFR) in ovarian cancer. Gynecol Oncol. 1997;65:36-41.

33. Clark S, Cheng DJ, Hsuan JJ, Haley JD, Waterfield MD. Loss of three major auto phosphorylation sites in the EGF receptor does not block the mitogenic action of EGF. J Cell Physiol. 1998;134:421-28.

34. Shamieh LS, Evans AJ, Denton MC, Clinton GM. Receptor binding specificities of Herstatin and its intron 8-encoded domain. FEBS Lett. 2004;568:163-66.

35. Azios NG, Romero FJ, Denton MC, Doherty JK, Clinton GM. Expression of herstatin, an autoinhibitor of HER2/ neu, inhibits transactivation of HER 3 by HER 2 and blocks EGF activation of the EGF receptor. Oncogene. 2001;20:5:199-209.

36. Jhabvala-Romero F, Evans A, Guo S, Denton M, Clinton GM. Herstatin inhibits heregulin-mediated breast cancer cell growth and overcomes tamoxifen resistance in breast cancer cells that overexpress HER2. Oncogene. 2003;22:8178-186.

37. Lee $\mathrm{H}$, Maihle NJ. Isolation and characterization of four alternate c-erbB 3 transcripts expressed in ovarian carcinomaderived cell lines and normal human tissues. Oncogene. 1998;16:3242-252.

38. Lee H, Akita RW, Sliwkowski MX, Maihle NJ. A naturally occurring secreted human ErbB3 receptor isoform inhibits heregulin-stimulated activation of ErbB2, ErbB3, and ErbB4. Cancer Res. 2001;61:4467-473.

39. Petch LA, Harris J, Raymond VW, Blasband A, Lee DC, Earp HS. A truncated, secreted form of the epidermal growth factor receptor is encoded by an alternatively spliced transcript in normal rat tissue. Mol Cell Biol. 1990;10:2973-982.

40. FlickingerTW, MaihleNJ, Kung H-J. An alternatively processed mRNA from the avian c-erbB gene encodes a soluble, truncated form of the receptor that can block ligand-dependent transformation. Mol Cell Biol. 1992;12:883-93.

41. Carpenter G. EGF receptor transactivation mediated by the proteolytic production of EGF-like agonists. Science's stke, 2000; Perspective, online manuscript.

42. Higashiyama S, Horikawa M, Yamada K, Ichino N, Nakano $\mathrm{N}$, Nakagawa T, et al. A novel brain-derived member of the epidermal growth factor family that interacts with ErbB3 and ErbB4. J Biochem. 1997;122:675-80.

43. Grünwald V, Hidalgo M. Developing inhibitors of the epidermal growth factor receptor for cancer treatment. J Natl Cancer Inst. 2003;95:851-67.

44. Anklesaria P, Teixidó J, Laiho M, Pierce JH, Greenberger JS, Massagué J. Cell-cell adhesion mediated by binding of membrane-anchored transforming growth factor alpha to epidermal growth factor receptors promotes cell proliferation. Proc Natl Acad Sci USA. 1990;87:3289-293.

45. Dobashi Y, Stern DF. Membrane-anchored forms of EGF stimulate focus formation and intercellular communication. Oncogene. 1991;6:1151-159. 
46. Dong J, Opresko LK, Dempsey P, Lauffenburger DA, Coffey RJ, Wiley HS. Metalloprotease-mediated ligand release regulates autocrine signaling through the epidermal growth factor receptor. Proc Natl Acad Sci USA. 1999;96:6235-240.

47. Issing WJ, Liebich C, Wustrow TP, Ullrich A. Coexpression of epidermal growth factor receptor and TGF-alpha and survival in Upper aerodigestive tract cancer. Anticancer Res. 1996; 16:283-88.

48. Mendelsohn J, Baselga J. The EGF receptor family as targets for cancer therapy. Oncogene. 2000;19:6550-565.

49. Cho HS, Mason K, Ramyar KX, Stanley AM, Gabelli SB, Denney DW Jr, et al. Structure of the extracellular region of HER2 alone and in complex with the Herceptin Fab. Nature. 2003;421:756-60.

50. Modjtahedi H, Moscatello DK, Box G, Green M, Shotton C, Lamb DJ, et al. Targeting of cells expressing wild-type EGFR and type-III mutant EGFR (EGFRvIII) by antiEGFR Mab ICR62: a two-pronged attack for tumour therapy. Int J Cancer. 2003;105:273-80.

51. Shawver LK, Slamon D, Ullrich A. Smart drugs: tyrosine kinase inhibitors in cancer therapy. Cancer Cell. 2002;1:117-23.

52. Levitzki A. Tyrosine kinases as targets for cancer therapy. Eur J Cancer. 2002;38(suppl 5):S11-18.

53. Moulder SL, Yakes FM, Bianco R, Simpson JF, Arteaga CL. Epidermal growth factor receptor (HER1) tyrosine kinase inhibitor ZD1839 (Iressa) inhibits HER2/neu (erbB2overexpressing breast cancer cells in vitro and in vivo. Cancer Res. 2001;61:8887-895.

54. Xia W, Mullin RJ, Keith BR, Liu LH, Ma H, Rusnak DW, et al. Anti-tumor activity of GW572016: a dual tyrosine kinase inhibitor blocks EGF activation of EGFR/erbB2 and downstream Erk1/2 and AKT pathways. Oncogene. 2002;21:6255-263.

55. Anido J, Matar P, Albanell J, Guzman M, Rojo F, Arribas J, et al. ZD1839, a specific epidermal growth factor receptor (EGFR) tyrosine kinase inhibitor, induces the formation of unactive EGFR/HER2 and EGFR/HER3 heterodimers and prevents heregulin signaling in HER2-overexpresssing breast cancer cells. Clin Cancer Res. 2003;9:1274-283.

56. Arteaga CL. EGF receptor as a therapeutic target: patient selection and mechanisms of resistance to receptor-targeted drugs. J Clin Oncol. 2003;21:289s-91s.

57. She QB, Solit D, Basso A, Moasser MM. Resistance to gefitinib in PTEN-null HER-overexpressing tumor cells can be overcome through restoration of PTEN function or pharmacologic modulation of constitutive phosphoatidylinositol 3'-kinase/Akt pathway signaling. Clin Cancer Res. 2003;9:4340-346.

58. Lu Y, Yu Q, Zhang J, Wang H, Koul D, McMurray JS, et al. Src family protein-tyrosine kinases alter the function of PTEN to regulate phosphatidylinositol 3-kinase/AKT cascades. J Biol Chem. 2003;278:40057-40066.
59. Bianco R, Shin I, Yakes FM, Basso A, Rosen N, Tsurutani J, et al. Loss of PTEN/MMAC1/TEP in EGF receptorexpressing tumor cells counteracts the antitumor action of EGFR tyrosine kinase inhibitors. Oncogene. 2003;22:2812-822.

60. Junttila TT, Laato M, VahlbergT, Söderström K-O, Visakorpi $\mathrm{T}$, Isola J, et al. Identification of patients with transitional cell carcinoma of the bladder overexpressing ErbB2, ErbB3, or specific ErbB4 isoforms: real-time reverse transcription-PCR analysis in estimation of ErbB receptor status from cancer patients. Clin Cancer Res. 2003;9:5346-357.

61. Mosensson Y, Yarden Y. Oncogenic growth factor receptors: implications for signal transduction therapy. Semin Cancer Biol. 2004;14:262-70.

62. Mahtouk K, Jourdan M, DeVos J, Herthog C, Fiol G, Jourdan E, et al. An inhibitor of the EGF receptor family blocks myeloma cell growth factor activity of HB-EGF and potentiates dexamethasone or anti-IL-6 antibodyinduced apoptosis. Blood. 2004;103:1829-837.

63. Pain B, Woods CM, Saez J, FlickingerT, Raines M, Peyrot $S$, et al. EGFR as a hemopoietic growth factor receptor: the c-erbB product is present in chicken erytrocytic progenitors and controls their self-renewal. Cell. 1991;65:37-46.

64. Hayman MJ, Meyer S, Martin F, Steinlein P, Beug H. Selfrenewal and differentiation of normal avian erythoid progenitor cells: regulatory roles of the TGFalfa/c-ErbB and SCF/c-kit receptors. Cell. 1993;74:157-69.

65. Gandrillon O, Schmidt U, Beug H, Samarut J. TGF-alpha cooperates with TGF-beta to induce the self-renewal of normal erythrocytic progenitors: evidence for an autocrine mechanism. EMBO J. 1999;18:2764-781.

66. von Linden M, Deiner EM, Dolznig H, Parren-vonAmelsvoort M, Hayman MJ, Mullner EW, et al. Leukemic transformation of normal murine erythroid progenitors: $v$ and c-ErbB act through signaling pathways activated by the EpoR and c-kit in stress erythropoiesis. Oncogene. 2001;20:3651-664.

67. Bühring HJ, Sures I, Jallal B, Weiss FU, Busch FW, Ludwig WD, et al. The receptor tyrosine kinase p185HER2 is expressed on a subset of B-lymphoid blasts from patients with acute lymphoblastic leukemia and chronic myelogenous leukemia. Blood. 1995;86:1916-923.

68. Potti A, Ganti AK, Koch M, Levitt R, Mehdi SA. Immunohistochemical identification of HER-2/neu overexpression and CD117 (c-kit) expression in multiple myeloma. Leuk Lymphoma. 2002;43:2427-430.

69. Pizzatti L, Sá LA, Souza JM, Abdelhay E. Altered protein profile in chronic myeloid leukemia chronic phase identified by a comparative proteomic study. Biochim Biophys Acta. 2006;1764:929-42.

70. Vinante F, Rigo A, Papini E, Cassatella MA, Pizzolo G. Heparin-binding epidermal growth factor-like growth factor/diphtheria toxin receptor expression by acute myeloid leukemia cells. Blood. 1999;93:1715-723. 
71. Freitas CS, Dalmau SR, Kovary K, Savino W. Epidermal growth factor modulates fetal thymocyte growth and differentiation. Dev Immunol. 1998;5:169-82.

72. Freitas CS, Dalmau SR, Savino W. Epidermal growth factor modulates fetal thymocyte growth and differentiation: partial reversal by insulin, mimicking by specific inhibitors of EGF receptor tyrosine kinase activity, and differential expression of CD45 phosphatase isotypes. J Immunol. 1998;161:3384-392.

73. Freitas CS, Moreira MA, Dalmau SR. The epidermal growth factor precursor-gene segment encoding the soluble cytokine is conserved and constitutively expressed by malignant T-cell lines. Anais do XX Congresso da Sociedade
Brasileira de Imunologia; 2001 incluir aqui o período de realização do Congresso; Salvador (BA), Brasil.

74. Padron Rios G, Freitas CS. Detection of transcripts for HER1 receptors to epidermal growth factor in human leukemias. Anais do XIII Congresso da Sociedade Brasileira de Biologia Celular, IX Simpósio Brasileiro de Matriz Extracelular, e IV International Symposium on Extracellular Matrix; 2006 26-30 julho; Búzios (RJ), Brasil; Seção: Cell Differentiation, resumo B.01 pag 41 .

75. Padron Rios G, Santos de Moraes C, Freitas CS. Expressão de receptores da família HER e seus ligantes em malignidades hematopoéticas humanas. II Jornada de Iniciação Científica do INCA; 2007 junho; Rio de Janeiro (RJ), Brasil.

\section{Abstract}

Receptors belonging to the HER (ErbB) family are essential to the development of organs and systems in general. Although their presence is ubiquitous in adult tissues, their over-expression or dysregulation is associated with poor prognosis in many types of tumors. Curiously, the absence of HER and their ligands in mature blood cells has been reported, suggesting a necessary down-regulation during hematopoieis. However, many reports point to their presence in hematological malignancies, suggesting that in the latter, the new drugs currently being developed for other types of cancer will also putatively contribute to the control of cell growth. Formerly known only as transmembrane signal transducers, HER receptors have recently been found in cell nuclei, indicating new signaling routes. Nuclear localization has been described as a prognostic factor in breast cancer, while the extension of this observation to hematological malignancies remains to be investigated.

Key words: EGF, HER, Cancer, Hematopoiesis, Tyrosine kinase inhibitors, Tyrphostins 